\title{
Qualitative and Quantitative Assessment of Common Weed Species in West Gondar Sesame Growing Area, Ethiopia
}

\author{
Asfaw Azanaw ${ }^{*}$, Yohannes Ebabuye ${ }^{2}$, Yimer Abeje ${ }^{3}$ \\ ${ }^{1}$ Plant Pathologist, Gondar Agricultural Research Center, Gondar, ETHIOPIA \\ ${ }^{2}$ Entomologist, Gondar Agricultural Research Center, Gondar, ETHIOPIA \\ ${ }^{3}$ Research Assistant, Gondar Agricultural Research Center, Gondar, ETHIOPIA
}

*Corresponding Contact:

Email: asfaw.azanaw@yahoo.com

Manuscript Received: 19 July 2020 - Revised: 25 August $2020 \quad$ - Accepted: 01 Sept 2020

\begin{abstract}
Weeds compete with crops grown for resource limits such as water, nutrients and light. Weed infestation is one of the major factors limiting sesame production, as its seedlings grow slowly during the first few weeks making it a weak competitor at earlier crop growth stages. Weed induced sesame yield reductions of up to 65 percent, and a need for a crucial weed-free duration of up to 50 days following planting. Weed surveys are useful for determining the occurrence and relative importance of weed species in crop production systems. Weed survey on farm basis is needed to establish efficient weed management and decision making mechanism and to evaluate weed control measures. Therefore this survey was conducted to assess qualitative and quantitative weed species in sesame growing areas of west Gondar, Ethiopia. Weed survey was conducted in 2012 at Metema and 2013 main cropping season at Metema, Tach Armachiho and Mirab Armachiho district data on weed species type and count were taken along the diagonal with $50 \mathrm{~cm} \times 50 \mathrm{~cm}$ quadrate at 15 meter interval at three points. Visual identification, manuals, photographs, leaf lets, and books were used for identifying weed species. They conducted interviews with farmers, development agents and district experts working in the region. Weed species compositions were analyzed by frequency, abundance, dominance and similarity index. In the districts 31common weed species or groups of species from 17 families were recorded. Ipomea cordofana Br., Grass spp', Commelina benghalensis L. Andropogon abyssinicus (Fresen) R. Br., Boerhaavia erecta L., Corchorus olitorius L.kudra, Corchorus trilocularis L., Leucas martinicensis (Jacq) ait.f. and Ipomea triloba are the most prevalent, abundant and important weeds in sesame fields in north Gondar. As to this on farm and practical training on identification and demonstration management options should be given on the low land of north Gondar farmers.
\end{abstract}

Keywords: Weed, Frequency, Abundance, Dominance and Similarity Index 


\section{INTRODUCTION}

Sesame is an annual plant belonging to the family Pedaliaceae. It is a short-day plant and is normally self-pollinated although cross-pollination occurs between 5 and over 50 percent (Pathirana, 1994). It is an erect annual herbaceous plant with two characteristics of growth: indeterminate and determinate, with heights of up to two meters. Most varieties show an indeterminate habit of growth, which is also shown as a continuous production of new leaves, flowers and capsules so long as the environment remains suitable for growth (Carlsson et al., 2008). The period of growth ranges from 70 to 150 days, depending on the variety and cultivation conditions (Ashri, 1998).

Weeds compete with crops grown for resource limits such as water, nutrients and light (Oudhia, 2004). Weeds infestation also causes disease issues, acts as an alternative habitat for deleterious insects and pathogens, delays harvesting operations, raises production costs, decreases crop market value and increases the risk of fire in perennial crops, plantations and forest reserves (Holm et al., 1977; Oudhia, 2004).

Weed infestation is one of the major factors limiting sesame production, as its seedlings are growing slowly during the first few weeks, making it a weak competitor at earlier crop growth stages (Grichar et al., 2011). Consequently, insufficient weed control during the early growth period of sesame can cause a significant reduction in yield (Narkheda et al., 2000). Kropff \& Spitters (1991) stated that, in a competitive situation, the key factor affecting sesame yield is the ratio between the relative leaf area of the weed and the crop at the time the crop canopy is closed. The impact on the establishment and development of sesame from weeds have been well known. Balyan (1993) and Singh et al. (1992) recorded weed-induced sesame yield reductions of up to 65 per cent and a crucial weed-free duration of up to 50 days following planting. Eagleton et al. (1987) estimated a weed biomass six times that of sesame 48 days after planting under weedy conditions and Bennett (1993) registered a weed biomass 1.3 fold that of sesame 42 days after planting.

Weed surveys are useful for determining the occurrence and relative importance of weed species in crop production systems (Frick \& Thomas, 1992; McCully et al., 1991). Weed survey on farm basis is needed to establish efficient weed management and decision making mechanism and to evaluate weed control measures. Besides, it is useful to record population changes of potentially dangerous weeds, to high light area where changes in species diversity occur, and to give guidelines for setting up research and extension priorities in weed control activities. This survey was therefore conducted to assess qualitative and quantitative weed species in sesame growing areas of north Gondar, Ethiopia.

\section{Materials AND Methods}

Two kinds of surveys can be done on fields; qualitative survey which involves simply determining weeds species. It does not indicate the infestation level or degree of economic importance of the weeds. Quantitative survey is more informative and better recognized than the qualitative determination as it reports characteristics or parameters which can be used to describe a weed community quantitatively.

\section{Intensity of weed species}

Weed survey was conducted in 2012 at Metema and 2013 main cropping season at Metema, Tach Armachiho and Mirab Armachiho district in North Gondar Zone Ethiopia. Sample fields were randomly assessed in the districts and data on weed species type and count were 
taken along the diagonal with $50 \mathrm{~cm} \times 50 \mathrm{~cm}$ quadrate at 15 meter interval at three points. For identification of weed species type visual identification, manuals, photographs, leaf lets and books were used. Interviewing with farmers, development agents and the districts experts working in the area were made. Weed species compositions were analyzed by frequency $(\mathrm{F})$, abundance (A), dominance (D) and similarity index (SI) determinations using the formulae described by (Taye and Yohannes, 1998).

Frequency $(\mathrm{F})$ is the percentage of sampling plots on which a particular weed species is found. It explains how often a weed species occurs in the survey area in which a species occurred in at least one quadrat (Thomas, 1985).

$\mathrm{F}=\mathrm{X} / \mathrm{N} \times 100$, where F-frequency, $\mathrm{X}$-number of occurrences of a weed species and Nsample number

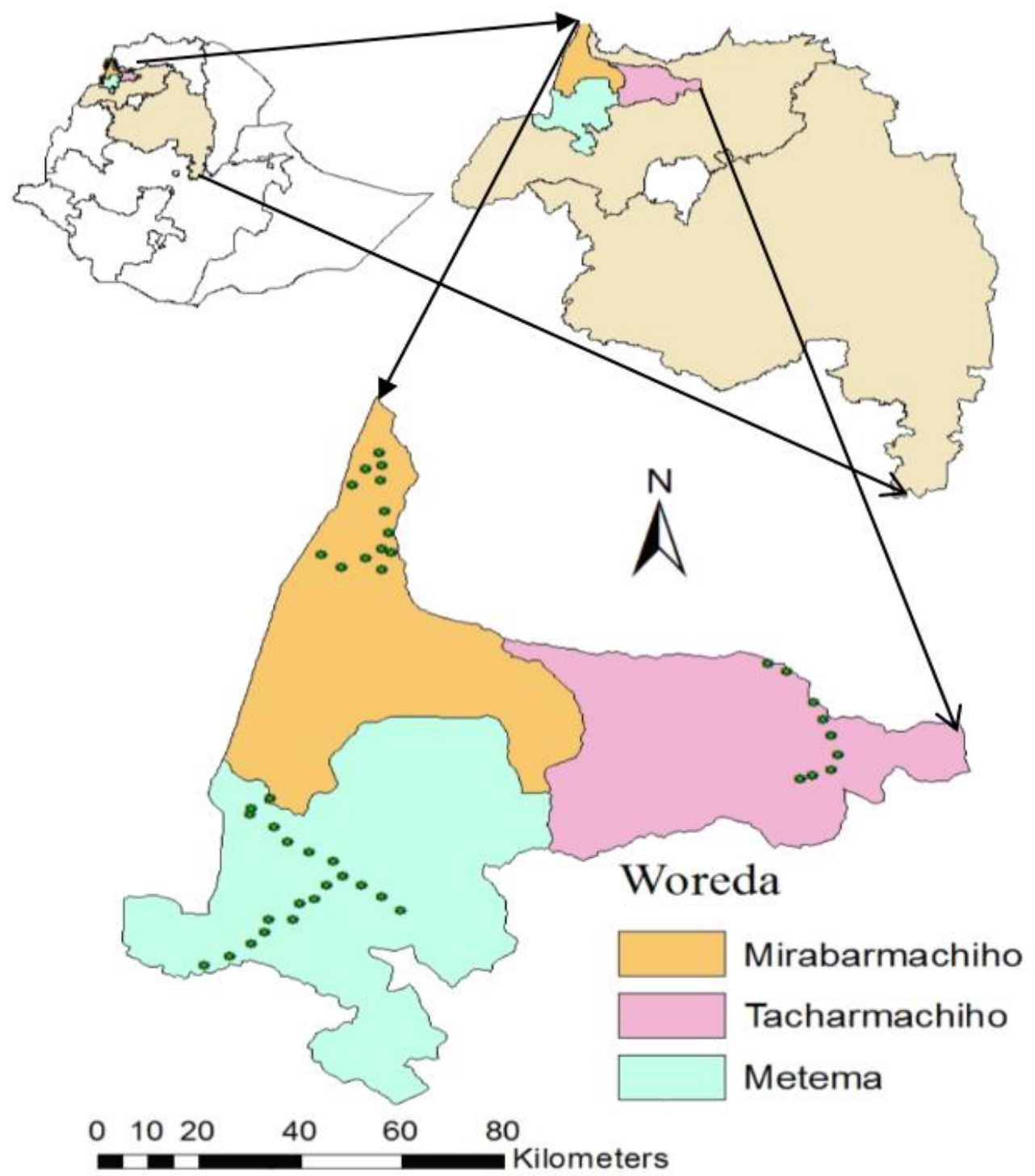




\section{The Study Areas}

North Gondar Zone is located at $11027^{\prime} 31^{\prime \prime}-13^{\circ} 46^{\prime} 10^{\prime \prime} \mathrm{N}$ latitude and $35016^{\prime} 41^{\prime \prime}-$ $38^{\circ} 46^{\prime} 30^{\prime \prime} \mathrm{E}$ longitude, within altitudes ranges of 978.4-1828.9 m.a.s.l. The annual average rain fall was $1115 \mathrm{~mm}$. The mean maximum and minimum temperature of the area is $100 \mathrm{C}$ and $42^{\circ} \mathrm{C}$, respectively.

Abundance (A) is population density of a weed species expressed as the number of individuals of weed plants per unit area.

$\mathrm{A}=\sum \mathrm{W} / \mathrm{N}$, where A-Abundance, $\mathrm{W}$-number of individuals of species and $\mathrm{N}$-sample number

Dominance (D) is abundance of an individual weed species in relation to total weed abundance.

$\mathrm{D}=\mathrm{A}^{*} 100 / \sum \mathrm{A}$, where D-Dominance, $\mathrm{A}$ - Abundance and $\sum \mathrm{A}$-total abundance

Similarity index (SI) is similarity of weed communities among different locations.

$\mathrm{SI}=100 \times \mathrm{E}_{\mathrm{pg}} /\left(\mathrm{E}_{\mathrm{pg}}+\mathrm{E}_{\mathrm{pa}}+\mathrm{E}_{\mathrm{pb}}\right)$, where, SI- Similarity index,

Epg-number of species found in both locations, Epa-number of species found in only locations I

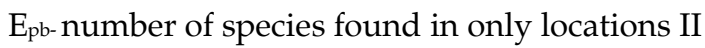

\section{Data Analysis}

The data collected were subjected to descriptive statistical analysis. The geographical distribution and intensity of weed species map was prepared using Computer Software (Archview, GIS) Version.

\section{RESULTS AND DISCUSSIONS}

Weed survey was done at Metema for two years in 2012 and 2013 and Armachiho were done in 2013 on the rainy season. Within cropping sites, 31common weed species or groups of species from 17 families were recorded, the most common being the Gramineae (7), Convolvulaceae (3), and 21 from other family. Vegetation assessment showed weed flora in the survey areas consisted of grassy and broad leaved weeds. Broad leaf types were the most dominant than narrow (grass) type of weeds and twenty seven of them were annual and Capparis tonantosa (lam), Acacia spp and Ziziphus spina Christi were perennial type of weeds (Table 1).

Table 1: Weeds leaf type and life cycle in low land of N. Gondar in 2012 and 2013 main cropping seasons

\begin{tabular}{|l|c|c|c|c|}
\hline Districts & \multicolumn{2}{|c|}{ Weed leaf type (\%) } & \multicolumn{2}{c|}{ Weed life cycle (\%) } \\
\hline & Broad & Narrow & Annual & Perennial \\
\hline Metema & 16 & 7 & 22 & 4 \\
\hline Tach Armachiho & 20 & 8 & 24 & 4 \\
\hline Mirab Armachiho & 21 & 8 & 26 & 4 \\
\hline
\end{tabular}

The similarity index of weed species in different cropping systems was determined using the method described by Ogbeibu in 2005 in Adesina et al. (2012) the critical level $=50 \%$ (i. e. $>50 \%=$ similar, $<50 \%=$ dissimilar). As to this the weeds in the three districts was similar was similar in type as critical level of more than fifty percent assessed in the cropping sites (Graph 1). 


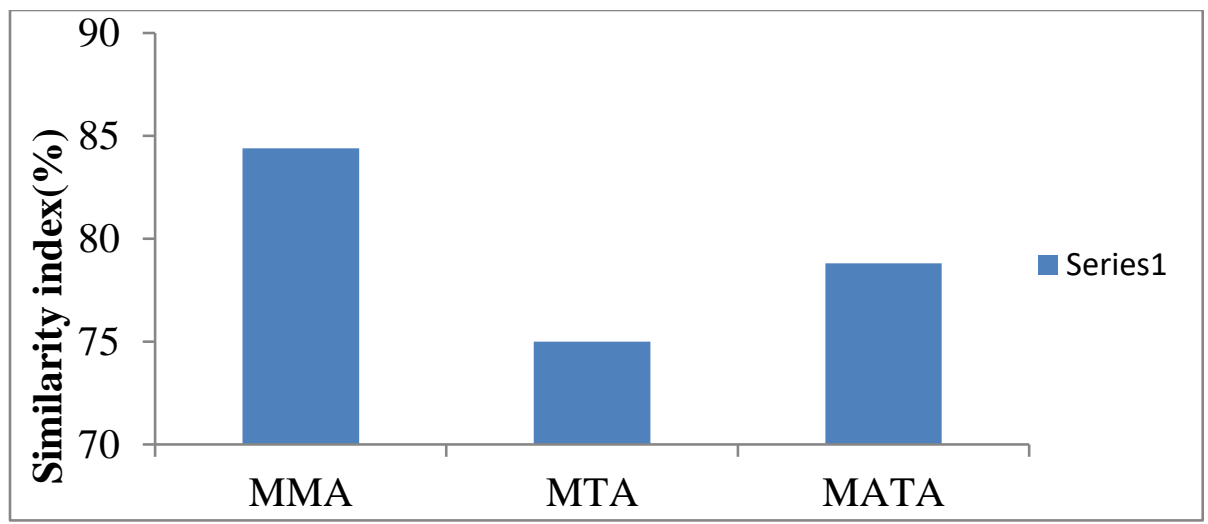

Graph 1. Similarity index of weeds among the districts in sesame fields for 2013 cropping season

NB: $M=$ Metema, MA=Mirab Armachiho and TA= Tach Armachiho

In sesame fields, weeds with frequency of Ipomea cordofana (60\%), 'Kok sar'(73.3\%), Phyllanthusniruri (66.6\%). Andropogon abyssinicus (73.3\%), and 'Awnda' (70\%) in 2012 cropping season and 'Kok sar'(85\%), Corchorus trilocularis(70\%), Ipomea triloba (65\%), Andropogon abyssinicus (60\%), and 'Awnda' (65\%) in 2013 cropping season were the most prevalent weeds at Metema while the rest weeds were less than $60 \%$ frequency in each sesame fields. At Tach armachiho, Commelina benghalensis (70\%), Andropogon abyssinicus (70\%), Kyllinga bulbosa (80\%) and Corchorus trilocularis $(78 \%)$ were the most prevalent weeds in sesame fields in 2013 cropping season. Ipomea cordofana (70\%), 'Kok sar'(65\%), 'Awnda' (70\%), Corchorus trilocularis (75\%) and 'Yelam Ayin'(85\%) were the most frequent weeds at Mirab armachiho in 2013 cropping seasons in sesame fields (Table 3, 4, and 5 ).

Table 2: Frequency, Abundance and Dominance of common weeds on sesame in 2012 at Metema in North Gondar

\begin{tabular}{|c|c|c|c|c|c|c|c|c|c|c|}
\hline \multirow{2}{*}{$\begin{array}{l}\text { Local } \\
\text { (Amharic)name }\end{array}$} & \multicolumn{2}{|c|}{ Weed species } & \multirow[t]{2}{*}{ Family } & \multicolumn{2}{|c|}{ Life cycle } & \multicolumn{2}{|c|}{ Leaf type } & \multirow[t]{2}{*}{$F(\%)$} & \multirow[t]{2}{*}{$\mathbf{A}$} & \multirow[t]{2}{*}{ D } \\
\hline & Common name & Scientific name & & & Pere. & broad & narrow & & & \\
\hline Dirya & Ipomea & $\begin{array}{c}\text { Ipomea } \\
\text { cordofana Br. }\end{array}$ & Convolvulaceae & $\mathrm{x}$ & & $\mathrm{x}$ & & 60 & 0.84 & 0.02 \\
\hline $\begin{array}{l}\text { Wuha } \\
\text { ankur }\end{array}$ & $\begin{array}{c}\text { Wondering } \\
\text { Jew }\end{array}$ & $\begin{array}{c}\text { Commelina } \\
\text { benghalensis L. }\end{array}$ & Commelinaceae & $x$ & & $\mathrm{x}$ & & 36.6 & 1.24 & 0.03 \\
\hline Kok sar & Grass spp. & Grass spp. & Gramineae & $\mathrm{x}$ & & & $\mathrm{x}$ & 73.3 & 4.82 & 0.10 \\
\hline- & Gripeweed & Phyllanthusniruri L. & & $\mathrm{x}$ & & & $\mathrm{x}$ & 66.6 & 1.48 & 0.03 \\
\hline Ras kimr & Bobbin weed & $\begin{array}{c}\text { Leucas martinicensis } \\
\text { (Jacq) ait.f. }\end{array}$ & Labiatea & $x$ & & $\mathrm{x}$ & & 46.6 & 2.58 & 0.06 \\
\hline Dememo & Foxtail grass & $\begin{array}{c}\text { Setaria pumila } \\
\text { Roem. and schult }\end{array}$ & Gramineae & $x$ & & & $x$ & 56.6 & 3.06 & 0.07 \\
\hline Zemen sar & & $\begin{array}{c}\text { Andropogon } \\
\text { abyssinicus R.Br. }\end{array}$ & Gramineae & $x$ & & & $x$ & 73.3 & 3.21 & 0.07 \\
\hline $\begin{array}{l}\text { Gid zemede } \\
\text { (Lekelek) }\end{array}$ & $\begin{array}{c}\text { Erect } \\
\text { Spiderling }\end{array}$ & $\begin{array}{c}\text { Boerhaavia } \\
\text { erecta } \mathrm{L} \text {. }\end{array}$ & Nyctaginaceae & $x$ & & & & 33.3 & 1.13 & 0.02 \\
\hline Kudra & & $\begin{array}{c}\text { Corchorus olitorius } \\
\text { L.kudra }\end{array}$ & Tiliaceae & $x$ & & $\mathrm{x}$ & & 33.3 & 0.23 & 0.01 \\
\hline Gicha & & $\begin{array}{l}\text { Kyllinga bulbosa } \\
\text { P.Beauv. }\end{array}$ & Cyperaceae & $\mathrm{x}$ & & & $\mathrm{x}$ & 40 & 2.83 & 0.06 \\
\hline- & Astma weed & Euphorbia hirta L. & Euphorbiaceae & & & & & 10 & 0.23 & 0.01 \\
\hline Amira & Hummera weed & $\begin{array}{c}\text { Corchorus } \\
\text { trilocularis L. }\end{array}$ & Tiliaceae & $x$ & & $\mathrm{x}$ & & 53.3 & 8.35 & 0.18 \\
\hline Hareg & Ipomia & Ipomia triloba & Convolvulaceae & $\mathrm{x}$ & & $x$ & & 36.6 & 0.18 & 0.004 \\
\hline
\end{tabular}


Azanaw et al.: Qualitative and Quantitative Assessment of Common Weed Species in West Gondar Sesame Grawing Area, Ethiopia

\begin{tabular}{|l|c|c|l|l|l|c|c|c|c|c|}
\hline Akakma & Puncture vine & Tribulus terrestris L. & Zygophyllaceae & $\mathrm{x}$ & & $\mathrm{x}$ & & 23.3 & 0.18 & 0.004 \\
\hline Yebeg lat & Purslane & Portulaca oleraceae L. & Portulacaceae & $\mathrm{x}$ & & $\mathrm{x}$ & & 23.3 & 0.49 & 0.01 \\
\hline Yehya abish & Siclepod & Senna obtusifolia & Caesalpiniaceae & $\mathrm{x}$ & & $\mathrm{x}$ & & 36.6 & 0.63 & 0.01 \\
\hline Nug ambesa & Dodder & Cuscuta campestris & Convolvulaceae & $\mathrm{x}$ & & & $\mathrm{x}$ & 6.6 & 0.03 & 0.001 \\
\hline $\begin{array}{l}\text { Yewsha } \\
\text { dagusa }\end{array}$ & $\begin{array}{c}\text { Wild finger } \\
\text { millet }\end{array}$ & Eleusine indica L. & Gramineae & $\mathrm{x}$ & & & $\mathrm{x}$ & 3.3 & 0.03 & 0.001 \\
\hline Starbur & Starbur & $\begin{array}{c}\text { Acanthospermum } \\
\text { hispidum }\end{array}$ & Compositae & $\mathrm{x}$ & & $\mathrm{x}$ & & 10 & 0.17 & 0.004 \\
\hline $\begin{array}{l}\text { Yemogn fikir } \\
\text { zemed begde) }\end{array}$ & $\begin{array}{c}\text { Heart leaf } \\
\text { Cocklebur }\end{array}$ & $\begin{array}{c}\text { Xanthium } \\
\text { strumarium } \text { L. }\end{array}$ & Asteraceae & $\mathrm{x}$ & & $\mathrm{x}$ & & 3.3 & 0.01 & 0.0002 \\
\hline Aluma & Pig weed & Amarantus spp. & Amaranthaceae & $\mathrm{x}$ & & $\mathrm{x}$ & & 10 & 0.13 & 0.003 \\
\hline Gumero & - & $\begin{array}{c}\text { Capparis } \\
\text { tonantosa (lam) }\end{array}$ & Capparidaceae & & $\mathrm{x}$ & $\mathrm{x}$ & & 6.6 & 0.02 & 0.001 \\
\hline Girar & - & Acacia spp & Mimmosoideae & & $\mathrm{x}$ & $\mathrm{x}$ & & 20 & 0.17 & 0.004 \\
\hline Kurkura & - & $\begin{array}{c}\text { Ziziphus spina } \\
\text { Christi }\end{array}$ & Rhamnaceae & & $\mathrm{x}$ & $\mathrm{x}$ & & $<5$ & & \\
\hline
\end{tabular}

\section{$\mathrm{F}=$ Frequency, $\mathrm{A}=\mathrm{Abundance}, \mathrm{D}=$ Dominance}

Table 3: Frequency, Abundance and Dominance of common weeds on sesame in 2013 at Metema in North Gondar

\begin{tabular}{|c|c|c|c|c|c|c|c|c|c|c|}
\hline \multirow{3}{*}{$\begin{array}{l}\text { Local } \\
\text { (Amharic) } \\
\text { name }\end{array}$} & \multicolumn{2}{|c|}{ Weed species } & \multirow[t]{3}{*}{ Family } & \multirow{2}{*}{\multicolumn{2}{|c|}{ Life cycle }} & \multirow{2}{*}{\multicolumn{2}{|c|}{ Leaf }} & \multirow{3}{*}{$\begin{array}{c}F \\
(\%)\end{array}$} & \multirow[t]{3}{*}{ A } & \multirow[t]{3}{*}{$\mathrm{D}$} \\
\hline & \multirow{2}{*}{ Common name } & \multirow{2}{*}{ Scientific name } & & & & & & & & \\
\hline & & & & Annual & Pere. & broa & arrow & & & \\
\hline Dirya & Ipomea & Ipomea cordofana Br. & Convolvulaceae & $x$ & & $x$ & & 35 & 1.67 & 3.91 \\
\hline Wuha ankur & Wondering Jew & $\begin{array}{c}\text { Commelina } \\
\text { benghalensis L. }\end{array}$ & Commelinaceae & $x$ & & $x$ & & 50 & 1.03 & 2.42 \\
\hline Kok sar & Grass spp & Grass spp. & Gramineae & $\mathrm{x}$ & & & $\mathrm{x}$ & 85 & 3.57 & 8.37 \\
\hline- & Gripeweed & Phyllanthusniruri L. & & $\mathrm{x}$ & & $x$ & & 54.6 & 1.12 & 2.62 \\
\hline Ras kimr & Bobbin weed & $\begin{array}{c}\text { Leucas martinicensis } \\
\text { (Jacq) ait.f. }\end{array}$ & Labiatea & $x$ & & $x$ & & 42 & 1.42 & 2.66 \\
\hline Hareg & Ipomea & Ipomea triloba & Convolvulaceae & $x$ & & $x$ & & 65 & 1.33 & 3.13 \\
\hline Dememo & Foxtail grass & $\begin{array}{c}\text { Setaria pumila Roem. } \\
\text { and schult }\end{array}$ & Gramineae & $\mathrm{x}$ & & & $\mathrm{x}$ & 40 & 1.12 & 2.62 \\
\hline Zemen sar & & $\begin{array}{c}\text { Andropogon } \\
\text { abyssinicus } \\
\text { (Fresen) R.Br. }\end{array}$ & Gramineae & $\mathrm{x}$ & & & $\mathrm{x}$ & 60 & 1.65 & 3.87 \\
\hline $\begin{array}{l}\text { Gid zemede } \\
\text { (Lekelek) }\end{array}$ & $\begin{array}{c}\text { Erect } \\
\text { Spiderling } \\
\end{array}$ & Boerhaavia erecta $L$. & Nyctaginaceae & $x$ & & $\mathrm{x}$ & & 40 & 2.45 & 5.75 \\
\hline Kudra & & $\begin{array}{c}\text { Corchorus olitorius } \\
\text { L.kudra }\end{array}$ & Tiliaceae & $x$ & & $\mathrm{x}$ & & 45 & 1.37 & 3.21 \\
\hline Gicha & & $\begin{array}{c}\text { Kyllinga bulbosa } \\
\text { P.Beauv. }\end{array}$ & Cyperaceae & $x$ & & & $\mathrm{x}$ & 20 & 0.47 & 1.10 \\
\hline- & Astma weed & Euphorbia hirta L. & Euphorbiaceae & & & $x$ & & 15 & 0.23 & 0.55 \\
\hline Amira & Hummera weed & $\begin{array}{c}\text { Corchorus trilocularis } \\
\text { L. }\end{array}$ & Tiliaceae & $x$ & & $x$ & & 70 & 1.48 & 3.48 \\
\hline Akakma & Puncture vine & Tribulus terrestris L. & Zygophyllaceae & $\mathrm{x}$ & & $\mathrm{x}$ & & 10 & 0.07 & 0.16 \\
\hline Yehya abish & Siclepod & Senna obtusifolia & Caesalpiniaceae & $x$ & & $\mathrm{x}$ & & 55 & 0.65 & 1.53 \\
\hline Nug ambesa & Dodder & Cuscuta campestris & Convolvulaceae & $x$ & & - & - & 10 & 0.1 & 0.2 \\
\hline Yebeg lat & Purslane & Portulaca oleraceae L. & Portulacaceae & $x$ & & $\mathrm{x}$ & & 23.3 & 0.49 & 0.01 \\
\hline $\begin{array}{l}\text { Yewsha } \\
\text { dagusa }\end{array}$ & $\begin{array}{c}\text { Wild finger } \\
\text { millet }\end{array}$ & Eleusine indica $L$. & Gramineae & $x$ & & & $\mathrm{x}$ & 20 & 0.28 & 0.66 \\
\hline Sitar ber & Starbur & $\begin{array}{l}\text { Acanthospermum } \\
\text { hispidum DC. }\end{array}$ & Compositae & $x$ & & $\mathrm{x}$ & & 5 & 0.20 & 0.47 \\
\hline $\begin{array}{l}\text { Yemogn fikir } \\
\text { / } \\
\text { zemed begde }\end{array}$ & $\begin{array}{l}\text { Heartleaf } \\
\text { Cocklebur }\end{array}$ & $\begin{array}{c}\text { Xanthium strumarium } \\
\text { L. }\end{array}$ & Asteraceae & $x$ & & $\mathrm{x}$ & & 5 & 0.17 & 0.39 \\
\hline Aluma & Pig weed & Amarantus spp. & Amaranthaceae & $\mathrm{x}$ & & $\mathrm{x}$ & & 5 & 0.02 & 0.04 \\
\hline Gumero & & $\begin{array}{c}\text { Capparis tonantosa } \\
\text { (lam) }\end{array}$ & Capparidaceae & & $x$ & $\mathrm{x}$ & & 6.6 & 0.02 & 0.001 \\
\hline Girar & & Acacia spp. & Mimmosoideae & & $\mathrm{x}$ & $\mathrm{x}$ & & 20 & 0.17 & 0.004 \\
\hline Kurkura & & Ziziphus spina Christi & Rhamnaceae & & $x$ & $x$ & & & & \\
\hline
\end{tabular}

$\mathrm{F}=$ Frequency, $\mathrm{A}=$ Abundance, $\mathrm{D}=$ Dominance 
Table 4: Frequency, Abundance and Dominance of common weeds on sesame in 2013 at Tach Armachiho in North Gondar

\begin{tabular}{|c|c|c|c|c|c|c|c|c|c|c|}
\hline \multirow{2}{*}{$\begin{array}{l}\text { Local } \\
\text { (Amharic) } \\
\text { name }\end{array}$} & \multicolumn{2}{|c|}{ Weed species } & \multirow[t]{2}{*}{ Family } & \multicolumn{2}{|c|}{ Life cycle } & \multicolumn{2}{|c|}{ Leaf } & \multirow{2}{*}{$\begin{array}{c}\mathrm{F} \\
(\%)\end{array}$} & \multirow[t]{2}{*}{$\mathrm{A}$} & \multirow[t]{2}{*}{$\mathrm{D}$} \\
\hline & Common name & Scientific name & & Annual & Pere. & broad & narrow & & & \\
\hline Dirya & Ipomea & Ipomea cordofana $\mathrm{Br}$. & Convolvulaceae & $x$ & & $\mathrm{x}$ & & 30 & 1.2 & 3.23 \\
\hline Wuha ankur & Wondering Jew & $\begin{array}{c}\text { Commelina benghalensis } \\
\text { L. }\end{array}$ & Commelinaceae & $\mathrm{x}$ & & $\mathrm{x}$ & & 70 & 1.87 & 5.03 \\
\hline Kok sar & Grass spp & Grass spp. & Gramineae & $\mathrm{x}$ & & & $\mathrm{x}$ & 40 & 0.47 & 1.26 \\
\hline Ras kimr & Bobbin weed & $\begin{array}{l}\text { Leucas martinicensis } \\
\text { (Jacq) ait.f. }\end{array}$ & Labiatea & $\mathrm{x}$ & & $\mathrm{x}$ & & 70 & 1.06 & 2.85 \\
\hline Hareg & Ipomea & Ipomea triloba & Convolvulaceae & $\mathrm{x}$ & & $\mathrm{x}$ & & 40 & 0.33 & 0.89 \\
\hline $\begin{array}{l}\text { Dememo } \\
\text { (Yewsha } \\
\text { sinde ) }\end{array}$ & Foxtail grass & $\begin{array}{l}\text { Setaria pumila (poir.) } \\
\text { Roem. and schult }\end{array}$ & Gramineae & $\mathrm{x}$ & & & $\mathrm{x}$ & 50 & 0.65 & 1.75 \\
\hline Zemen sar & & $\begin{array}{c}\text { Andropogon abyssinicus } \\
\text { R.Br. }\end{array}$ & Gramineae & $\mathrm{x}$ & & & $\mathrm{x}$ & 70 & 1.73 & 4.66 \\
\hline $\begin{array}{l}\text { Gid zemede } \\
\text { (Lekelek) }\end{array}$ & $\begin{array}{c}\text { Erect } \\
\text { Spiderling }\end{array}$ & Boerhaavia erecta $\mathrm{L}$. & Nyctaginaceae & $x$ & & $\mathrm{x}$ & & 50 & 0.57 & 1.53 \\
\hline Kudra & --- & $\begin{array}{c}\text { Corchorus olitorius } \\
\text { L.kudra }\end{array}$ & Tiliaceae & $\mathrm{x}$ & & $\mathrm{x}$ & & 10 & 0.2 & 0.54 \\
\hline Gicha & & Kyllinga bulbosa P.Beauv. & Cyperaceae & $\mathrm{x}$ & & & $\mathrm{x}$ & 80 & 10.9 & 29.3 \\
\hline- & Astma weed & Euphorbia hirta L. & Euphorbiaceae & $x$ & & $\mathrm{x}$ & & 10 & 0.07 & 0.19 \\
\hline Amira & Hummera weed & Corchorus trilocularis & Tiliaceae & $\mathrm{x}$ & & $\mathrm{x}$ & & 78 & 10.4 & 27.9 \\
\hline Akakma & Puncture vine & Tribulus terrestris L. & Zygophyllaceae & $\mathrm{x}$ & & $\mathrm{x}$ & & 30 & 0.93 & 2.50 \\
\hline Yehya abish & Siclepod & Senna obtusifolia & Caesalpiniaceae & $\mathrm{x}$ & & $\mathrm{x}$ & & 40 & 0.23 & 0.62 \\
\hline Nug ambesa & Dodder & Cuscuta campestris & Convolvulaceae & $x$ & & - & - & 20 & 0.5 & 1.46 \\
\hline $\begin{array}{l}\text { Yewsha } \\
\text { dagusa }\end{array}$ & $\begin{array}{l}\text { Wild finger } \\
\text { millet }\end{array}$ & Eleusine indica L. & Gramineae & $\mathrm{x}$ & & & $\mathrm{x}$ & 20 & 4.16 & 11.2 \\
\hline Sitar ber & Starbur & $\begin{array}{c}\text { Acanthospermum } \\
\text { hispidum }\end{array}$ & Compositae & $x$ & & $\mathrm{x}$ & & 10 & 0.3 & 0.81 \\
\hline Aluma & Pig weed & Amarantus spp. & Amaranthaceae & $\mathrm{x}$ & & $\mathrm{x}$ & & 5 & 0.02 & 0.04 \\
\hline Gumero & & Capparis tonantosa (lam) & Capparidaceae & & $\mathrm{x}$ & $\mathrm{x}$ & & $<5$ & & \\
\hline Girar & & Acacia spp & Mimmosoideae & & $x$ & $\mathrm{x}$ & & $<5$ & & \\
\hline Kurkura & & Ziziphus spina Christi & Rhamnaceae & & $\mathrm{x}$ & $\mathrm{x}$ & & $<5$ & & \\
\hline Muja & - & Snowdenia polystachya & Gramineae & $\mathrm{x}$ & & & $\mathrm{x}$ & 40 & 0.63 & 1.70 \\
\hline $\begin{array}{l}\text { Yeseyitan } \\
\text { merfie }\end{array}$ & Black jack & Bidens pilosa & Compositae & $\mathrm{x}$ & & $\mathrm{x}$ & & 20 & 0.34 & 0.91 \\
\hline $\begin{array}{l}\text { Yeberha } \\
\text { Serdo }\end{array}$ & Bermuda grass & Cynodon dactylon & Gramineae & $x$ & & & $\mathrm{x}$ & 80 & 2.97 & 7.99 \\
\hline & & & & & & & & & & \\
\hline
\end{tabular}

\section{$\mathrm{F}=$ Frequency, $\mathrm{A}=\mathrm{Abundance}, \mathrm{D}=$ Dominance}

Table 5: Frequency, Abundance and Dominance of common weeds on sesame in 2013 at Mirab Armachiho in North Gondar

\begin{tabular}{|c|c|c|c|c|c|c|c|c|c|c|}
\hline \multirow{3}{*}{$\begin{array}{l}\text { Local } \\
\text { (Amharic) } \\
\text { name }\end{array}$} & \multicolumn{2}{|c|}{ Weed species } & \multirow[t]{3}{*}{ Family } & \multirow{2}{*}{\multicolumn{2}{|c|}{ Life cycle }} & \multirow{2}{*}{\multicolumn{2}{|c|}{ Leaf type }} & \multirow{3}{*}{$\begin{array}{c}\mathrm{F} \\
(\%)\end{array}$} & \multirow[t]{3}{*}{$\mathbf{A}$} & \multirow[t]{3}{*}{$\mathbf{D}$} \\
\hline & \multirow{2}{*}{$\begin{array}{l}\text { Common } \\
\text { name }\end{array}$} & \multirow[t]{2}{*}{ Scientific name } & & & & & & & & \\
\hline & & & & Annual & Pere. & broad & narrow & & & \\
\hline Dirya & Ipomea & Ipomea cordofana Br. & Convolvulaceae & $\mathrm{x}$ & & $x$ & & 70 & 3.02 & 9.25 \\
\hline Wuha ankur & $\begin{array}{c}\text { Wondering } \\
\text { Jew }\end{array}$ & $\begin{array}{c}\text { Commelina benghalensis } \\
\text { L. }\end{array}$ & Commelinaceae & $\mathrm{x}$ & & $\mathrm{x}$ & & 25 & 0.14 & 0.41 \\
\hline Kok sar & - & Grass spp. & Gramineae & $\mathrm{x}$ & & & $\mathrm{x}$ & 65 & 1.85 & 5.67 \\
\hline Ras kimr & Bobbin weed & $\begin{array}{l}\text { Leucas martinicensis } \\
\text { (Jacq) ait.f. }\end{array}$ & Labiatea & $\mathrm{x}$ & & $\mathrm{x}$ & & 5 & 0.07 & 0.20 \\
\hline Hareg & Ipomea & Ipomea triloba & Convolvulaceae & $\mathrm{x}$ & & $\mathrm{x}$ & & 13.3 & 0.09 & 0.002 \\
\hline $\begin{array}{l}\text { Dememo } \\
\text { (Yewsha } \\
\text { sinde) }\end{array}$ & \begin{tabular}{|l|} 
Foxtail grass \\
\end{tabular} & $\begin{array}{l}\text { Setaria pumila (poir.) } \\
\text { Roem. and schult }\end{array}$ & Gramineae & $\mathrm{x}$ & & & $x$ & 56.6 & 3.06 & 0.07 \\
\hline Awnda & & & & $\mathrm{x}$ & & $\mathrm{x}$ & & 70 & 11.3 & 0.24 \\
\hline Zemen sar & & Andropogon abyssinicus & Gramineae & $x$ & & & $\mathrm{x}$ & 15 & 0.13 & 0.41 \\
\hline
\end{tabular}




\begin{tabular}{|c|c|c|c|c|c|c|c|c|c|c|}
\hline & & (Fresen) R.Br. & & & & & & & & \\
\hline $\begin{array}{l}\text { Gid zemede } \\
\text { (Lekelek) }\end{array}$ & $\begin{array}{c}\text { Erect } \\
\text { Spiderling }\end{array}$ & Boerhaavia erecta $L$. & Nyctaginaceae & $\mathrm{x}$ & & $\mathrm{x}$ & & 25 & 1.3 & 3.99 \\
\hline Kudra & & $\begin{array}{c}\text { Corchorus olitorius } \\
\text { L.kudra }\end{array}$ & Tiliaceae & $\mathrm{x}$ & & $\mathrm{x}$ & & 10 & 0.28 & 0.87 \\
\hline Gicha & & Kyllinga bulbosa P.Beauv. & Cyperaceae & $\mathrm{x}$ & & & $\mathrm{x}$ & 5 & 0.03 & 0.10 \\
\hline- & Astma weed & Euphorbia hirta L. & Euphorbiaceae & $\mathrm{x}$ & & $\mathrm{x}$ & & 10 & 0.23 & 0.01 \\
\hline Amira & $\begin{array}{l}\text { Hummera } \\
\text { weed }\end{array}$ & Corchorus trilocularis L. & Tiliaceae & $\mathrm{x}$ & & $\mathrm{x}$ & & 75 & 2.92 & 8.94 \\
\hline Gorjijit & & & & $x$ & & & & 33.3 & 0.28 & 0.01 \\
\hline Akakma & Puncture vine & Tribulus terrestris L. & Zygophyllaceae & $x$ & & $\mathrm{x}$ & & 5 & 0.1 & 0.31 \\
\hline Yehya abish & Siclepod & Senna obtusifolia & Caesalpiniaceae & $\mathrm{x}$ & & $x$ & & 10 & 0.7 & 2.15 \\
\hline $\begin{array}{l}\text { Gagrda } \\
\text { (Mechera) }\end{array}$ & & & & $\mathrm{x}$ & & & $x ?$ & 56.6 & 1.76 & 0.04 \\
\hline Nug ambesa & Dodder & Cuscuta campestris & Convolvulaceae & $\mathrm{x}$ & & & - & 4 & 0.1 & 0.3 \\
\hline $\begin{array}{l}\text { Yewsha } \\
\text { dagusa }\end{array}$ & $\begin{array}{c}\text { Wild finger } \\
\text { millet }\end{array}$ & Eleusine indica $L$. & Gramineae & $\mathrm{x}$ & & & $\mathrm{x}$ & 10 & 0.37 & 1.12 \\
\hline Sitar ber & Starbur & $\begin{array}{c}\text { Acanthospermum } \\
\text { hispidum }\end{array}$ & Compositae & $\mathrm{x}$ & & $\mathrm{x}$ & & 10 & 0.17 & 0.004 \\
\hline $\begin{array}{l}\text { Yemogn fikir } \\
\text { zemed begde }\end{array}$ & $\begin{array}{l}\text { Heart leaf } \\
\text { Cocklebur }\end{array}$ & Xanthium strumarium L. & Asteraceae & $\mathrm{x}$ & & $\mathrm{x}$ & & 3.3 & 0.01 & 0.0002 \\
\hline Yelam Ayin & $\begin{array}{c}\text { Yellow } \\
\text { wood sorrel }\end{array}$ & Oxalis spp. & & $\mathrm{x}$ & & $\mathrm{x}$ & & 85 & 12.8 & 39.14 \\
\hline Aluma & Pig weed & Amarantus spp. & Amaranthaceae & $\mathrm{x}$ & & $\mathrm{x}$ & & 10 & 0.13 & 0.003 \\
\hline Gumero & & Capparis tonantosa (lam) & Capparidaceae & & $\mathrm{x}$ & $\mathrm{x}$ & & 6.6 & 0.02 & 0.001 \\
\hline Girar & & Acacia spp & Mimmosoideae & & $\mathrm{x}$ & $\mathrm{x}$ & & 5 & 0.05 & 0.15 \\
\hline Kurkura & & Ziziphus spina Christi & Rhamnaceae & & $\mathrm{x}$ & $\mathrm{x}$ & & 5 & $\begin{array}{c}0.01 \\
7\end{array}$ & 0.05 \\
\hline Gimel wayika & --- & --- & ----- & $\mathrm{x}$ & & $\mathrm{x}$ & & 45 & 0.73 & 2.25 \\
\hline Muja & & Snowdenia polystachya & Gramineae & $\mathrm{x}$ & & & $\mathrm{x}$ & 25 & 0.43 & 1.33 \\
\hline Bolenta & & & & $\mathrm{x}$ & & $\mathrm{x}$ & & 70 & 1.1 & 3.37 \\
\hline Jib shinkurt & & & & $x$ & & $x$ & & 10 & 0.12 & 0.36 \\
\hline Chewchewit & & $\begin{array}{c}\text { Dinebra retroflexa (vahl.) } \\
\text { panzer }\end{array}$ & Gramineae & $x$ & & & $\mathrm{x}$ & 30 & 0.57 & 1.74 \\
\hline
\end{tabular}

$\mathrm{F}=$ Frequency, $\mathrm{A}=$ Abundance, $\mathrm{D}=$ Dominance

The Relative Densities or abundances of the various weed species are shown in (Table 3-5). Weed with relative abundance, 'Kok sar' (4.82), Leucas martinicensis (2.58), Setaria pumila (3.06), 'Awnda'(11.31), Andropogon abyssinicus (3.21), Kyllinga bulbosa (2.83), and Corchorus trilocularis (8.35), were the most densely grown weeds in sesame fields in 2012 at Metema. 'Kok sar' (3.57), Boerhaavia erecta (2.45) and Corchorus trilocularis (8.35) were emerged the most abundant weeds in sesame fields in 2013 at Metema district. At Tach armachiho, Ipomea cordofana (1.2), Commelina benghalensis (1.87), Leucas martinicensis (1.06), Andropogon abyssinicus (1.73), Kyllinga bulbosa (10.9), Corchorus trilocularis (10.4), Eleusine indica (4.16) and Cynodon dactylon (2.97) were the most abundant weeds in 2013 cropping season. Ipomea cordofana (3.02), 'Grass spp (1.85), Boerhaavia erecta (1.3), and Corchorus trilocularis (2.92) were also the most densely grown weeds in sesame fields at Mirab armachiho.

Weeds those with high dominancy Grass spp (0.1), Corchorus trilocularis (0.18), were very important in sesame fields in 2012 at Metema and in 2013 Ipomea cordofana (3.91), 'Koksar'(8.37), Ipomea triloba (3.13), Andropogon abyssinicu (3.87), Boerhaavia erecta (5.75), Corchorus olitorius (3.21), and Corchorus trilocularis (3.48) were dominant at Metema. Ipomea cordofana (3.23), Commelina benghalensis (5.03), Andropogon abyssinicus (4.66), Kyllinga bulbosa (29.3), Corchorus trilocularis (27.9), Eleusine indica (11.2) and Cynodon dactylon (7.99) were important weeds at Tach armachiho in 2013 cropping season. Ipomea cordofana (9.25), Grass spp (5.67), Boerhaavia erecta (3.99), and Corchorus trilocularis (8.94) were the most dominant weeds at Mirab armachiho. 


\section{Conclusions and Recommendations}

This survey has assessed the most abundant and troublesome weed species in sesame field in north Gondar. 31 common weed species or groups of species from 17 families are identified and quantified. Ipomea cordofana Br., Grass spp, Commelina benghalensis L. Andropogon abyssinicus (Fresen) R.Br., Boerhaavia erecta L., Corchorus olitorius L.kudra, Corchorus trilocularis L., Leucas martinicensis (Jacq) ait.f. and Ipomea triloba are the most prevalent, abundant and important weeds in sesame fields in north Gondar. Information that is vital for setting research and development priorities concerning sesame weed identification and management is developed. Furthermore, it has documented the current distribution and importance of the recently grown sesame weed species. As to this on farm and practical training on identification and demonstration management options should be given on the low land of north Gondar farmers.

\section{ACKNOWLEDGMENT}

We would like to express our appreciation to Amhara Regional Agricultural Research Institute (ARARI) and PPPO Project for its financial support. We are also indebted for the assistances provided by the research and technical staff of the pulse and oil case team of Gondar Agricultural Research Center (GARC).

\section{REFERENCES}

Adesina, G. O., Akinyemiju, O. A., \& Ola, O. T. (2012). Assessment of frequency, density and abundance of weed species in different Cropping Systems. Journal of Natural Sciences Research Vol.2, No.9, pp.107-119

Ashri, A. (1998). Sesame breeding. Plant Breed. rev. 16, 179-228.

Balyan, R. (1993). Integrated weed management in oilseed crops in India. Proc. India Sump, Indian Soc. Weed Sci. 1, 317-323.

Bennett, M. (1993). Sesame Research Report 1991-92. Wet season, Katherine. Northern Territory, Australian Dept. of Primary Industries and Fisheries, Technical Bulletin No. 215.

Carlsson, A. S., Chanana, N. P., Gudu, S., Suh M. C., \& Were, B. A. (2008). Sesame. In: Kole, C., et al. (Eds.) Compendium of transgenic crop plant- Transgenic oilseed crops. pp. 227-246. Texas, USA: Wiley Blackwell; 2. ISBN 978-1-405-16924-0.Center), Mexico.

Eagleton, G., Sandover, S., \& Dickson, M. (1987). Research Report: Sesame Seed 1982-1986. Dept. Agric. Kununurra, West Australia.

Frick, B., \& Thomas A. G. (1992). Weed surveys in different tillage systems in southwestern Ontario field crops. Can. J. Plant Sci., 72:1337-1347.

Grichar, J. W., David, C. S., Kevind, B., Brent, A. B., \& Charles, R. S. (2011). Sesame (Sesamum indicum L) tolerance and weed control with soil applied herbicides. Crop protection 20, 389-394

Holm, I. G., Plucknett, D. L., Pancho, J. V., \& Herberger, J. P., (1997). The world worst weeds; Distribution and biology,Honolulu University press of hawai, $609 \mathrm{pp}$.

Kropff, M. \& Spitters, C. (1991). A simple model of crop loss by weed competition from early observations on relative leaf area of weeds. Weed Res. 31, 97-105.

McCully, K.V., Sampson, M. G., \& Watson, A. K. (1991). Weed survey of Nova Scotia lowbush blueberry (Vaccinium angustifolium) fields. Weed Sci. 29:180-185

Narkheda, T. N., Wadile, S. C., Attarde, D. R; Suryawanghi, R. T. (2000). Integrated weed management in sesame (Sesamum indicum L) under rainfed condition. Indian Journal Agricultural Research 34, 247 - 250. 
Ogbeibu, A. E. (2005). Biostatistics: A Practical Approach to Research and Data Handling. Mindex Publishing Company Limited, Benin City.

Oudhia, P. (2004). Phyto-sociological studies of rainy wasterlands weed with special reference to Parthenrium hysterophous L. IN Repair District http://www.iprng.org/IPRNG - and w 15. Htm

Pathirana, R. (1994). Natural cross-pollination in sesame (Sesamum indicum L.). Plant Breed. 112(2), 167170.

Singh, D., Dagar, J., and Gangwar, B. (1992). Infestation by weeds and their management in oilseed crops - a review. Agric. Rev. 13, 163-175.

Taye, T. \& Yohannes, L. (1998). Qualitative and quantitative determination of weeds in teff in west Shewa Zone. Arem 4: 46-60.

Thomas, A. G. (1985). Weed survey system used in Saskatchewan for cereal and oilseed crops. Weed Sci. 33:34-43.

\section{How to cite this article}

Ambaw, A., Ebabuye, Y., \& Abeje, Y. (2020). Qualitative and Quantitative Assessment of Common Weed Species in West Gondar Sesame Growing Area, Ethiopia. ABC Journal of Advanced Research, 9(2), 53-62. https://doi.org/10.18034/abcjar.v9i2.507 